\title{
Benefit from a high dose of gefitinib in a woman with lung adenocarcinoma and an epidermal growth factor receptor tyrosine-kinase acquired mutation
}

\author{
EDITTA BALDINI $^{1}$, TIZIANA PROCHILO ${ }^{1}$, LAURA BOLDRINI ${ }^{2}$, \\ FRANCA MELFI $^{3}$ and GABRIELLA FONTANINI ${ }^{2}$ \\ Dipartimenti di ${ }^{1}$ Oncologia, ${ }^{2}$ Chirurgia, ${ }^{3}$ Cardio-Toracico, Azienda Ospedaliero-Universitaria Pisana, Pisa, Italy
}

Received October 29, 2007; Accepted November 27, 2007

\begin{abstract}
Over the last 10 years, the approach to the treatment of cancer has changed significantly due to an improved understanding of the processes that regulate tumor growth and development. Targeted strategies act against various neoplasms, alone or in combination with conventional therapy, while avoiding most of the toxicities induced by standard chemotherapy and radiotherapy. Promising targets include members of the human epidermal receptor (HER) family, such as epidermal growth factor receptor (HER1/EGFR). Many EGFR-targeted agents, especially tyrosine-kinase inhibitors (TKIs), are being developed for the treatment of advanced non-small cell lung cancer (NSCLC) in which EGFR shows an activating mutation in the tyrosine-kinase domain. Mutations seem to be clinically relevant as they are most frequent in nonsmokers, adenocarcinomas, women and Japanese patients, features related to a radiographic response to TKIs. Here, we report the management of a stage IV adenocarcinoma of the lung in a young non-smoking woman treated with gefitinib whose tumor developed EGFR-TKI resistance.
\end{abstract}

\section{Introduction}

Gefitinib was the first oral EGFR-TK inhibitor introduced in the second/third-line of treatment for advanced non-small cell lung cancer (NSCLC) based on results obtained from two large phase II trials (IDEAL-1 and IDEAL-2) $(1,2)$. These phase II studies showed that $250 \mathrm{mg}$ of gefitinib was as effective as $500 \mathrm{mg}$ in an unselected patient population, although the latter was not the maximum tolerated dose. Subsequently, two large randomized phase III trials, testing the efficacy of gefitinib in combination with standard chemotherapy, were carried out on previously untreated NSCLC patients $(3,4)$. Unfortunately,

Correspondence to: Dr Editta Baldini, Oncology Department, S. Chiara University Hospital, Via Roma 67, I-56132 Pisa, Italy

E-mail: e.baldini@med.unipi.it

Key words: gefitinib, lung adenocarcinoma, epidermal growth factor receptor-tyrosine kinase neither of these trials demonstrated any survival advantages associated with the addition of gefitinib (whatever the dose) to conventional chemotherapy. In March 2005, the results of a phase III study (ISEL) comparing gefitinib with a placebo showed no survival advantage for this TKI (5). All the studies were carried out on unselected patients.

Despite the consistently observed low response rate, most patients experienced clinical benefit and an encouraging median rate of survival. This fostered retrospective studies to investigate predictive markers of response and to identify subgroups of patients more likely to benefit from gefitinib treatment. These analyses showed that a radiographic response to TKIs was more commonly reported in adenocarcinomas, non-smokers, women and Japanese patients $(6,7)$. Corresponding activating mutations in the tyrosine-kinase domain of EGFR were demonstrated (8), and these mutations were often reported in the same subgroups (9).

We now know that EGFR-TK mutations are determinants for gefitinib sensitivity. Unfortunately, nearly all patients who experience a clinical benefit from gefitinib actually progress during therapy. The potential mechanism of 'acquired' or 'secondary' resistance is a very important subject, with crucial clinical implications, and requires further investigation.

\section{Case report}

In February 2005, a 44-year-old woman underwent a lumpectomy and sentinel lymph node procedure for a nodule in the upper part of the right breast. Histologic examination showed a poorly differentiated adenocarcinoma (maximum diameter, $0.8 \mathrm{~mm}$ ) with a negative sentinel node, negative hormonal receptors and negative HER2/neu and Ki67=56\%. A chest $\mathrm{X}$-ray and abdominal ultrasound were performed as part of preoperative tumor staging and a bone scan was scheduled. Tumor markers (CEA and CA15.3) were negative, and biochemistry only showed an alkaline phosphatase level above the upper normal range. Since the chest X-ray performed preoperatively seemed to show a modest irregularity of the right mediastinal profile, a CT scan was also performed, which showed enlargement of the mediastinal nodes and a small lung nodule (maximum diameter, $2 \mathrm{~cm}$ ) in the right upper lobe. Moreover, a bone scan revealed hot-spots in the 5 th right and 8th left ribs, and in some vertebral bodies (T10, L1, L2 and 


\section{Case}

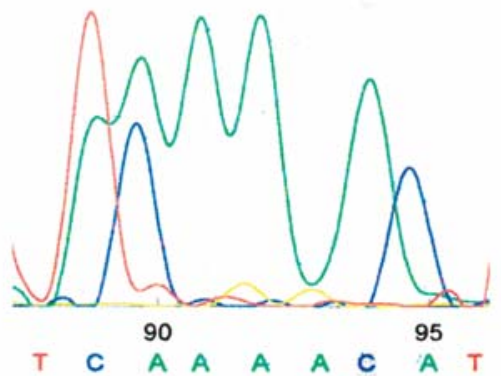

\section{Wild-type control}

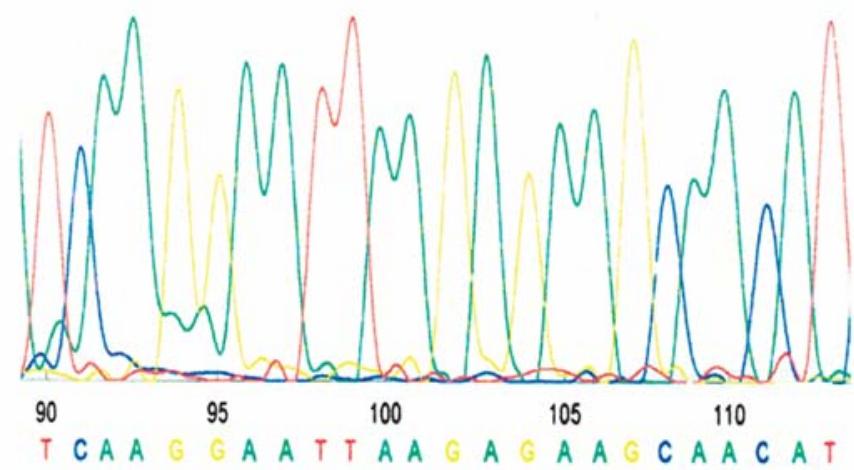

15 bp deletion (2235-2249)

Figure 1. Mutation analysis of EGFR. Automated sequencing of the EGFR gene in our patient, with a heterozygous in-frame deletion in exon 19 within the tyrosine-kinase domain compared to a wild-type control specimen.

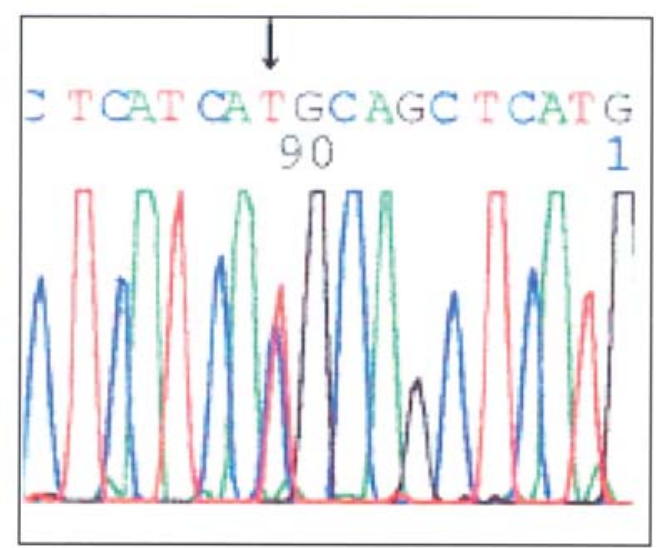

Figure 2. Automated sequencing of the EGFR gene, exon 20, of our patient. The mutant nucleotide (T) was present (T790M, ACG to ATG).

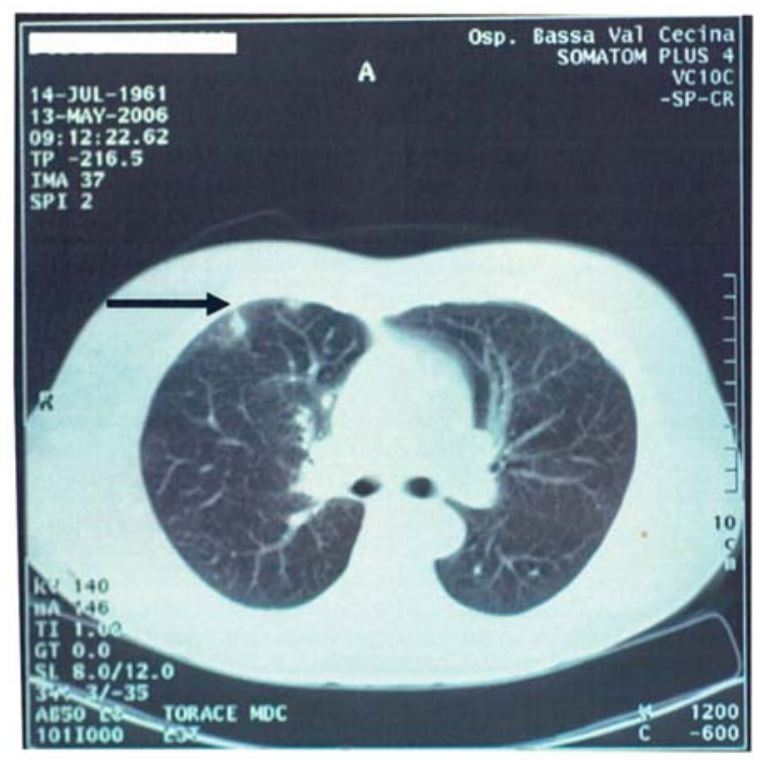

A
L3). Radiographic evaluation of these sites was negative, but a magnetic resonance of the spine confirmed the presence of osteoblastic lesions in L2 and L3.

A surgical procedure, during which the patient underwent a superior right lobectomy with mediastinal dissection and a biopsy of the 5th right rib, was necessary. Histology showed a poorly differentiated adenocarcinoma with metastases in 4 out of 10 mediastinal nodes. The bone lesion was a metastasis from the adenocarcinoma. A histological revision of all of the available material (breast nodule, lung tumor and bone biopsy) was required; immunohistochemistry revealed all specimens to be intensely positive for TTF1. The final diagnosis was of adenocarcinoma of the lung, with breast and bone metastases.

The patient, a female with no history of smoking and stage IV adenocarcinoma of the lung (the bone being the only remaining site of disease), came to our Medical Oncology Department. Determination of the EGFR-TK domain and

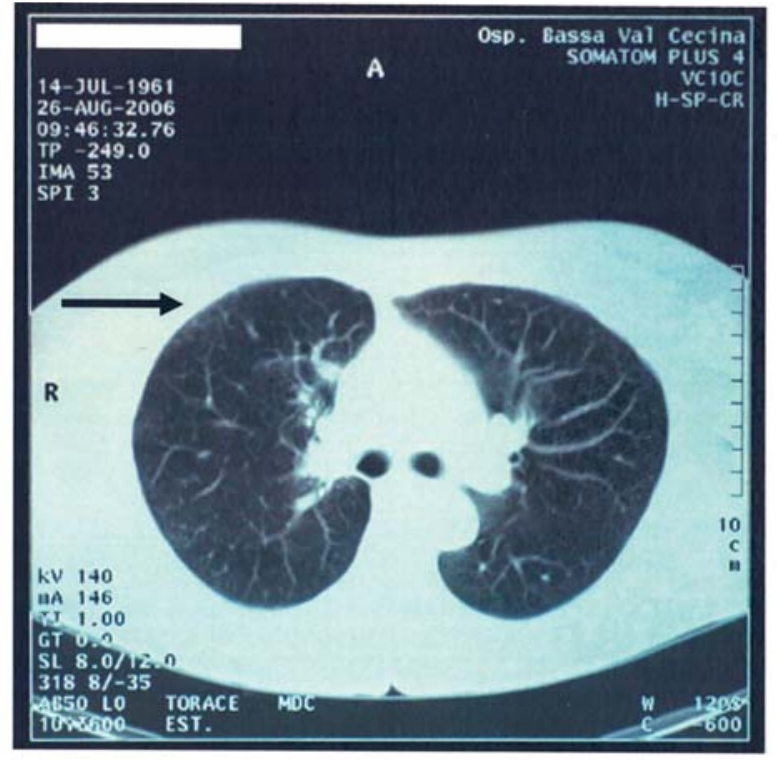

B

Figure 3. CT scan at progression (A) and 3 months after doubling the dose of gefitinib (B). 
K-ras mutations was performed using molecular techniques. The patient showed a mutation in exon 19, consisting of the deletion of 15 nucleotides from 2235 to 2249 (DEL E746A750), and wild-type (WT) K-ras (Fig. 1). The patient refused chemotherapy, and gefitinib $250 \mathrm{mg} /$ day was started as firstline therapy in March 2005. A cutaneous rush, limited to the face, developed two weeks later but was successfully treated with tetracycline and progressively disappeared during treatment. Six months from the onset of therapy, a bone scan showed no appearance of new bone lesions and a significant reduction in staining intensity of the pre-existent hot-spots. No disease progression was detected until May 2006 (14 months after starting gefitinib), when a single lymphadenopathy appeared in the right supraclavicular fossae. A fine-needle aspiration confirmed metastatic involvement and total re-staging also showed the presence of a small lung nodule (maximum diameter, $1 \mathrm{~cm}$ ) at the site of the previous intervention (CT scan). Mutation analysis of the EGFR-TK domain and K-ras was performed on lymph node specimens. The new molecular determinations showed a WT K-ras, the previously described mutation in exon 19 and the appearance of a new EGFR mutation in exon 20, which resulted in a threonine-to-methionine amino acid change at position 790 (T790M) of the kinase domain (Fig. 2).

The patient still refused chemotherapy but, aware of the new molecular results, decided to begin treatment with gefitinib at $500 \mathrm{mg} /$ day. Three months after doubling the dose of the drug, the right supraclavicular adenopathy disappeared and the small lung nodule was no longer evident (Fig. 3). Treatment is still in progress.

\section{Discussion}

Increasing knowledge of tumor biology is leading to more selective treatments. It is quite likely that, in the near future, the choice of the best therapeutic strategy will be guided, stepby-step, by molecular and genetic tests in each patient. Data from gastrointestinal stromal tumors and CML leukaemia clearly show that successful targeted treatments require the presence of the 'target' responsible for tumor growth. Therapy of this type will be applicable to and effective on small and very select subgroups of patients.

The inconsistent results obtained in NSCLC with TKIs were from studies performed on unselected patients; only a limited number of these patients strongly benefited from the drugs while hundreds did not, with a negative dilution effect on the overall results. Retrospective analyses of randomized trials largely suggest that tumors from patients who respond to TKIs usually harbour somatic gain-of-function mutations in the tyrosine-kinase domain. The most frequent activating mutations in the EGFR-TK domain are deletions in exon 19 (60\%), missense mutations in exon 21 (L858R) (25\%) and point mutations in exons 18, 20 and 21 (10).

Some types of mutations, on the other hand, seem to induce resistance to TKIs. In particular, K-ras mutations have been associated with primary resistance to these molecules (11). In addition, most patients initially sensitive to TKIs eventually relapse by developing secondary or acquired resistance to these drugs. Pao et al retrospectively examined the status of EGFR-TK exons 18-24 in tumor specimens from five patients who initially responded but, subsequently, progressed during treatment with TKIs (12). They showed that, in two out of five patients, progressive tumors had a second mutation in exon 20 which consisted of the substitution of threonine for methionine at position 790 in the catalytic cleft of the EGFR tyrosine-kinase domain, with consequent low-affinity binding of the TK inhibitor.

We observed the appearance of the same mutation in our patient during treatment, and comparison of the DNA sequences from the original tumor material and the second biopsy specimen, after 14 months of therapy with gefitinib, revealed the presence of a new peak at position 790 . In this female patient, the acquired resistance induced by the T790M mutation, overcome by doubling the dose of gefitinib, is of clinical interest. While not widely applicable, this type of therapeutic program, driven by the changing molecular profile of the patient's tumor, yielded relevant long-term results with a parallel preservation of quality of life.

\section{Acknowledgements}

This study was supported by AIDA $^{\mathrm{CP}}$ (Associazione Italiana Donne anti Cancro Polmonare).

\section{References}

1. Fukuoka M, Yano S, Giaccone G, et al: Multi-institutional randomized phase II trial of gefitinib for previously treated patients with advanced non-small cell lung cancer. J Clin Oncol 21: 2237-2246, 2003 .

2. Kris M, Natale RB, Herbst R, et al: Efficacy of gefitinib, an inhibitor of the epidermal growth factor receptor tyrosine kinase, in symptomatic patients with non-small cell lung cancer. JAMA 290: 2149-2158, 2003.

3. Giaccone G, Herbst RS, Manegold C, et al: Gefitinib in combination with gemcitabine and cisplatin in advanced non-small-cell lung cancer: a phase III trial - INTACT 1. J Clin Oncol 22: 777-784, 2004.

4. Herbst RS, Giaccone G, Schiller JH, et al: Gefitinib in combination with paclitaxel and carboplatinin in advanced non-smallcell lung cancer: a phase III trial - INTACT 2. J Clin Oncol 22: 785-794, 2004.

5. Thatcher N, Chang A, Parikh P, et al: Results of a phase III placebo-controlled study (ISEL) of gefitinib (IRESSA) plus best supportive care (BSC) in patients with advanced non-small-cell lung cancer (NSCLC) who had received 1 or 2 prior chemotherapy regimens. Lancet 366: 1527-1537, 2005.

6. Miller VA, Kris MG, Shah N, et al: Bronchioloalveolar pathologic subtype and smoking history predict sensitivity to gefitinib in advanced non-small-cell lung cancer. J Clin Oncol 22: 1103-1109, 2004.

7. Janne PA, Gurubhagavatula S, Yeap BY, et al: Outcomes of patients with advanced non-small-cell lung cancer treated with gefitinib (ZD 1839, 'Iressa') on an expanded access study. Lung Cancer 44: 221-230, 2004.

8. Lynch TJ, Bell DW, Sordella R, et al: Activating mutations in the epidermal growth factor receptor underlying responsiveness of non-small-cell lung cancer to gefitinib. N Engl J Med 350: 2129-2139, 2004.

9. Paez JG, Janne PA, Lee JC, et al: EGFR mutations in lung cancer: correlations with clinical response to gefitinib therapy. Science 304: 1497-1500, 2004.

10. Jänne PA: Ongoing first-line studies of epidermal growth factor receptor tyrosine kinase inhibitors in select patient populations. Semin Oncol 32 (Suppl 10): 9-15, 2005.

11. Pao W, Wang TY, Riely GJ, et al: KRAS mutations and primary resistance of lung adenocarcinomas to gefitinib or erlotinib. PLoS Med 2: e17, 2005.

12. Pao W, Miller VA, Politi KA, et al: Acquired resistance of lung adenocarcinomas to gefitinib or erlotinib is associated with a second mutation in the EGFR kinase domain. PLoS Med 2: e73, 2005. 